\title{
Electron bunch structure in energy recovery linac with high-voltage dc photoelectron gun
}

\author{
Y. M. Saveliev, ${ }^{*}$ F. Jackson, J. K. Jones, and J. W. McKenzie \\ ASTeC and Cockcroft Institute, Sci-Tech Daresbury, STFC, \\ Keckwick Lane, Daresbury, Warrington WA4 4AD, United Kingdom
}

(Received 10 June 2016; published 23 September 2016)

\begin{abstract}
The internal structure of electron bunches generated in an injector line with a dc photoelectron gun is investigated. Experiments were conducted on the ALICE (accelerators and lasers in combined experiments) energy recovery linac at Daresbury Laboratory. At a relatively low dc gun voltage of $230 \mathrm{kV}$, the bunch normally consisted of two beamlets with different electron energies, as well as transverse and longitudinal characteristics. The beamlets are formed at the head and the tail of the bunch. At a higher gun voltage of $325 \mathrm{kV}$, the beam substructure is much less pronounced and could be observed only at nonoptimal injector settings. Experiments and computer simulations demonstrated that the bunch structure develops during the initial beam acceleration in the superconducting rf booster cavity and can be alleviated either by increasing the gun voltage to the highest possible level or by controlling the beam acceleration from the gun voltage in the first accelerating structure.
\end{abstract}

DOI: 10.1103/PhysRevAccelBeams.19.094002

\section{INTRODUCTION}

Short, picosecond-range, electron bunches generated by photoinjector-based accelerators are not always of the highest quality, as might be expected. Some internal structure within the beam is often observed-the "hummingbird" beam image from JLab's IR free-electron laser (FEL) may serve as a striking example of such nonGaussian electron beams [1-3]. Physical mechanisms governing the beam formation in the injector are among various factors that may cause electron beams to become non-Gaussian. This should be especially relevant to injectors with dc photoelectron guns that operate at voltages below $500 \mathrm{kV}$, and this is the topic of this investigation.

Experimental studies of the structure of electron bunches were conducted on the ALICE (accelerators and lasers in combined experiments) energy recovery linac [4-6], where the beam is provided by a dc high-voltage (HV) photoelectron gun. The beam is initially focused, both transversely and longitudinally, using two solenoids and an rf $1.3 \mathrm{GHz}$ single-cell buncher. The beam is then accelerated in the superconducting (SC) rf booster to $6.5 \mathrm{MeV}$ and transported to the main SC rf linac, via the injector beam line shown in Fig. 1. From the linac, the beam emerges at 26.0 MeV energy, is transported to the magnetic compression chicane to enable the operation of the IR FEL [7] and the generation of broadband coherent terahertz radiation

*yuri.saveliev@stfc.ac.uk

Published by the American Physical Society under the terms of the Creative Commons Attribution 3.0 License. Further distribution of this work must maintain attribution to the author $(s)$ and the published article's title, journal citation, and DOI.
[6], and then is returned back to the main linac for energy recovery, before being dumped at $6.5 \mathrm{MeV}$.

Initially, the ALICE photoelectron gun was operated at a lower accelerating voltage of $230 \mathrm{kV}$, compared to the design value of $350 \mathrm{kV}$, due to hardware limitations. Some transverse beam structure was evident at the exit from the booster consisting of a tightly focused central part of the beam, the "core," and a larger "halo" [Fig. 2(a)]. This can be seen from two single-pixel beam profiles taken first through the center of the beam and second with a vertical offset [Fig. 2(b)]. In a dispersive section of the injector, the energy spectra normally exhibited two distinct beamlets with different beam energies [Fig. 2(d)]. The irregular shape of the beam images after the main linac suggests that this structure propagates all around the machine [Fig. 2(c)]. After installation of a larger gun HV ceramic and successful HV conditioning, the ALICE gun was capable of operating at up to $325 \mathrm{kV}$, thus making possible further beam investigations at higher voltages.

In this paper, we present the results of investigations into the causes of this beam structure, at the gun voltage of both 230 and $325 \mathrm{kV}$, particularly concentrating on the observation of two distinct beamlets in the injector (see Fig. 2).

\section{EXPERIMENTAL SETUP AND METHODS}

The booster cryogenic module consists of two ninecell TESLA-type $1.3 \mathrm{GHz}$ superconducting accelerating cavities. In standard injector setups, the first booster cavity (BC1) gradient is set to achieve $4.0 \mathrm{MeV}$ beam energy at the exit from $\mathrm{BC} 1$, while the second cavity (BC2) accelerates the beam further to $6.5 \mathrm{MeV}$. In typical ALICE regimes of operation, the off-crest phases are from $-15^{\circ}$ to $-10^{\circ}$ for $\mathrm{BC} 1$ and from $+10^{\circ}$ to $+25^{\circ}$ for $\mathrm{BC} 2$ (phases are 


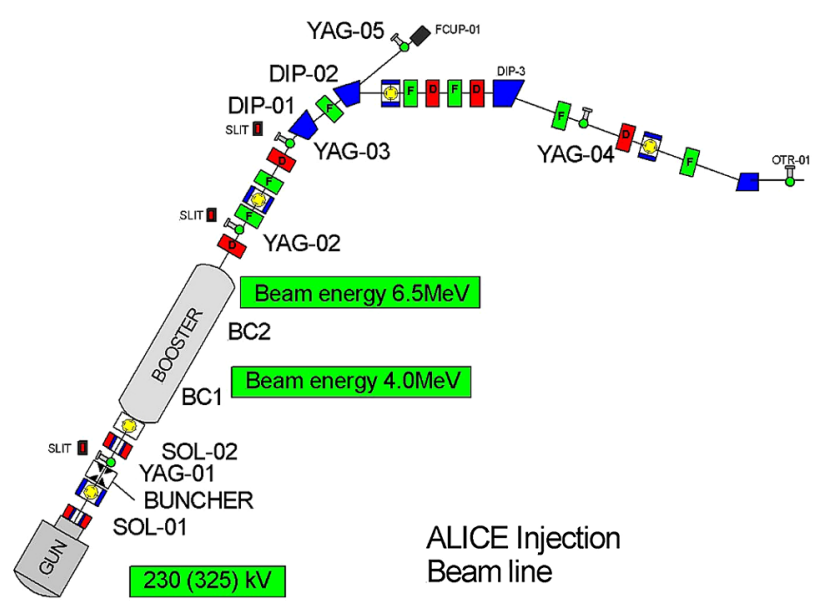

FIG. 1. Layout of the ALICE injector beam line. Diagnostic units with YAG-02 and YAG-03 contain also vertical slits on motorized stages. DIP-01 and screen YAG-05 constitute an energy spectrometer.

quoted with respect to wave crests and in terms of phase delays applied to the rf wave).

The experiments presented in this paper were conducted at a bunch charge of $\sim 50 \mathrm{pC}$, with the $\mathrm{BC} 1$ phase set to $-10^{\circ}$ and $\mathrm{BC} 2$ normally set to the zero-cross phase at $+90^{\circ}$. The energy difference between the beamlets of the electron bunches was determined either from a known dispersion of $1.2 \mathrm{~m}$ on YAG-05 or from the DIP-01 current difference, if both beamlets could not be seen on the screen simultaneously.

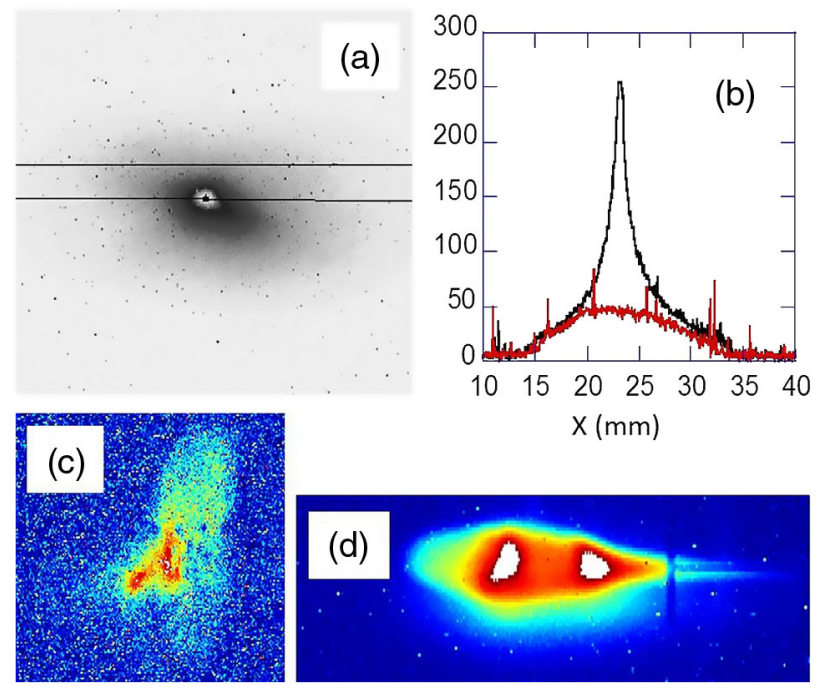

FIG. 2. (a) Beam image at the exit from the booster on the YAG-02 screen; (b) single-pixel horizontal profiles acquired along two horizontal lines shown in (a) - one through the center of the beam (black line) and with an offset from the center (red line); (c) beam image on one of the postlinac OTR screens; (d) beam image on the YAG-05 screen in a dispersive section of the ALICE injector.
The buncher was always set to a zero-cross phase using a time-of-arrival (TOA) diagnostic. The buncher peak voltage $V_{b}$ is controlled by the $\mathrm{rf}$ forward power $P_{b}$ in accordance with the relation $V_{b}=1.586 \sqrt{P_{b}}$ and where the units are $\mathrm{kV}$ and watts, respectively. This relation was initially validated by energy spectrometer measurements in a dedicated gun beam line [8] and later confirmed by TOA measurements on the full machine. For a given bunch charge and gun voltage, the buncher voltage is normally set to optimal values to ensure the required longitudinal properties of the bunch at the exit of the injector. In the course of these experiments, however, nonoptimal buncher settings were also used to "exaggerate" the nonGaussian bunch structure, thus facilitating more in-depth investigations.

Two diagnostic units in the injector beam line, YAG-02 and YAG-03, contain movable slits of $100 \mu \mathrm{m}$ width. These were used for beam emittance measurements and for blocking parts of the beam with the slit mechanical assemblies while observing the beam images on downstream screens.

Computer simulations suggest that, despite a relatively low beam energy in the injector, the longitudinal properties of the electron bunch (such as the bunch length and energy spread) are largely determined by $\mathrm{BC} 1$, with $\mathrm{BC} 2$ having a much smaller effect-controlling only the energy chirp of the bunch. This allows the use of $\mathrm{BC} 2$ as a diagnostic streaking cavity. For example, setting $\mathrm{BC} 2$ to a zero-cross phase of $\pm 90^{\circ}$ and varying the $\mathrm{BC} 2$ gradient changes the energy chirp of the bunch but keeps the mean electron bunch energy constant. The typical development of the bunch length and the energy spread from the cathode to the YAG-05 screen of the energy spectrometer, with BC2 set to a zero-cross phase, is illustrated in Fig. 3 using simulations performed in the space-charge code ASTRA [9]. The electron bunch is generated on the photocathode with a 28 ps FWHM laser pulse that is a combination of four fundamental Gaussian 7 ps FWHM laser pulses shifted in time with respect to each other in an optical pulse stacker. The bunch emerges from the gun with a positive energy chirp (electrons at the head of the bunch are of higher energy). The rf $1.3 \mathrm{GHz}$ buncher rotates the energy chirp to a negative value for longitudinal velocity bunching in the downstream beam line. Upon arrival at the entrance of $\mathrm{BC} 1$, the bunch experiences a large phase slippage due to its low initial beam energy from the gun and undergoes violent transformations in its longitudinal phase space. The mean beam energy initially decreases in the first cell of $\mathrm{BC} 1$ until the bunch phase slips to the accelerating part of the $\mathrm{rf}$ waveform. Independent of the $\mathrm{BC} 1$ phase, the bunch always exits $\mathrm{BC} 1$ with a positive energy chirp. If $\mathrm{BC} 2$ is set to $+90^{\circ}$, as in Fig. 3, it "imprints" an additional negative chirp on the bunch, with an amplitude directly proportional to the $\mathrm{BC} 2$ gradient. With a sufficiently high $\mathrm{BC} 2$ gradient, the energy chirp can be rotated from positive to negative, 

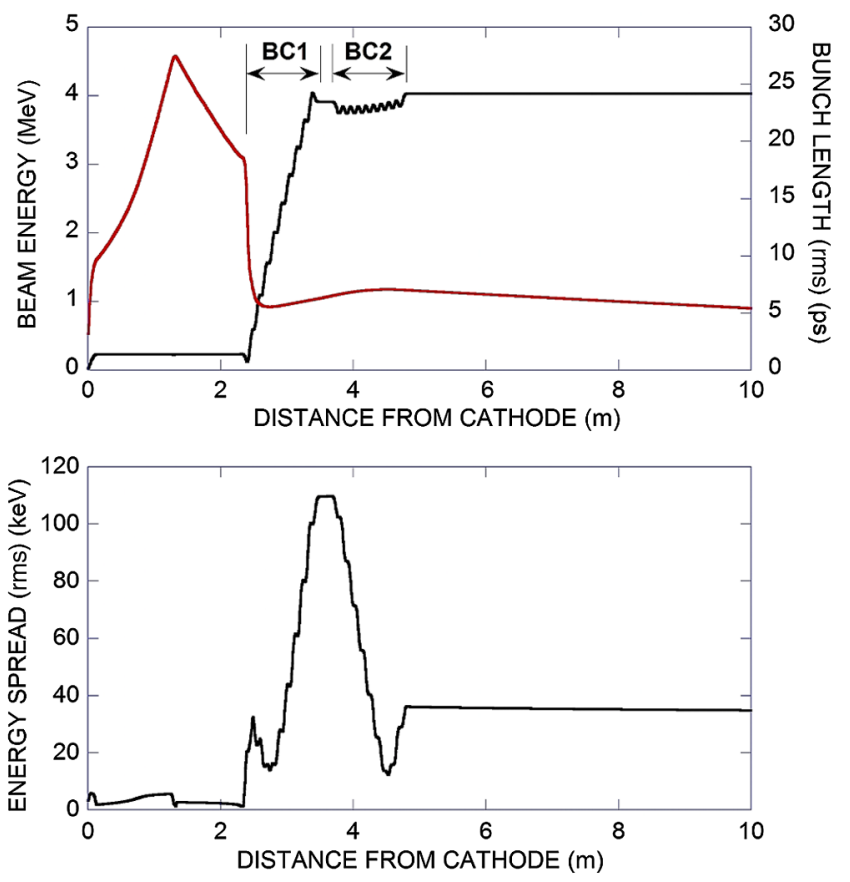

FIG. 3. Simulated (ASTRA) development of the mean beam energy (top, black line), the rms bunch length (top, red line), and the bunch energy spread (bottom) from the photoinjector cathode to the YAG-05 screen of the energy spectrometer. The first cavity (BC1) is set to $-10^{\circ}$ off-crest phase. The BC2 cavity is set to $+90^{\circ}$ zero-cross phase. The $\mathrm{BC} 2$ gradient is chosen such that the longitudinal phase space is rotated to the negative energy chirp at the exit from BC2. The gun voltage is $230 \mathrm{kV}$, and the final beam energy is $4.0 \mathrm{MeV}$. The buncher voltage is $31.7 \mathrm{kV}$.

and this case is also illustrated in Fig. 3. The resulting energy spread is observed in the energy spectrometer line, consisting of a dipole magnet DIP-01 and screen YAG05 (Fig. 1).

In simulations (Fig. 3), even with the low beam energy of 4.0 MeV, the energy spread does not change significantly from the exit of the booster to the screen of the energy spectrometer, located $\sim 10 \mathrm{~m}$ from the gun cathode. Simulations show also some noticeable longitudinal compression of the bunch that occurs due to the negative energy chirp, resulting in longitudinal velocity bunching. This simulated beam behavior appears to be in contradiction with some experimental observations, e.g., Ref. [10], where the longitudinal space charge (LSC) had a significant effect on the longitudinal emittance even at higher beam energies. We note, however, that our experiments were conducted at the relatively low bunch charge of $\sim 50 \mathrm{pC}$ and at longer bunch lengths. The latter is most important, because the LSC effect on the energy spread is inversely proportional to the square of the bunch length [11]. Using an analytical equation for the longitudinally Gaussian bunch [11], the effect of the LSC on the bunch energy spread can be estimated as $\sim 1 \mathrm{keV} / \mathrm{m}$. This estimation is not fully applicable, however, because, as will be shown later in
Sec. V, the simulated longitudinal bunch distribution is non-Gaussian and has a quasitriangular shape with a long tail instead. An analytical estimate of velocity bunching gives an $\sim 2$ ps reduction in the rms bunch length over $\sim 5 \mathrm{~m}$ from the booster exit that corresponds to ASTRA simulations in Fig. 3. Estimates for LSC bunch lengthening from the analytical model of a uniformly charged cylinder $[12,13]$ indicate a smaller by a factor of $2-3$ effect that should be considered as a guidance only because of the very different longitudinal distribution of the bunch charge. A detailed discussion of the validity of simulated bunch behavior in the drift space between the booster and the energy spectrometer (Fig. 3) is outside the scope of this paper, but the above crude analytical estimates provide some confidence in the validity of the experimental methodology employed in these experiments.

The simulated "rigidity" of the energy spread downstream of BC2 was also corroborated experimentally. The beam energy of $4.0 \mathrm{MeV}$ was fixed at the exit from $\mathrm{BC} 1$. The $\mathrm{BC} 2$ gradient was also fixed, and hence the negative energy chirp introduced by $\mathrm{BC} 2$ was constant, but the phase was varied $\pm 17^{\circ}$ around the zero-cross phase to generate a beam with $3.0,4.0$, and $5.0 \mathrm{MeV}$ energy at the exit of $\mathrm{BC} 2$. The energy separation between the two beamlets, clearly identifiable on the energy spectrum [similar to that in Fig. 2(d)], was not affected by the beam energy variation to within $\sim 10 \%$ of the accuracy of the measurements. The energy spread of individual beamlets decreases by $\sim 20 \%$, while the beam energy changes from 3.0 to $5.0 \mathrm{MeV}$, but, given the nature of these studies, such a beam evolution does not compromise the major results and conclusions.

\section{ELECTRON BEAM STRUCTURE AT 230 KV GUN VOLTAGE}

The energy separation between two peaks (beamlets) in the beam energy spectra depends on various parameters of the injector, most strongly on the buncher voltage. This is illustrated by Fig. 4, where the beam images on the energy spectrometer screen YAG-05 and corresponding energy spectra are given for different buncher voltages $V_{b}$. These data were collected at a dc gun voltage of $230 \mathrm{kV}$ and at a near-nominal injector setup, with $\mathrm{BC} 1$ and $\mathrm{BC} 2$ phases of $-10^{\circ}$ and $+40^{\circ}$, respectively. At low values of $V_{b}$, up to three peaks can be distinguished in the energy spectrum. At buncher voltages of $\sim 40 \mathrm{kV}$, corresponding to the minimum bunch length from the booster, we see the peaks merge together. At higher values of $V_{b}$ above $43 \mathrm{keV}$, the separation between peaks reappears. The peaks are always positioned at the high and the low ends of the energy spectra and thus determine an approximate FWHM energy spread of the electron bunch.

The full set of data from measurements (partially illustrated in Fig. 4) is presented in Fig. 5, where the energy separation between peaks in the energy spectra is 

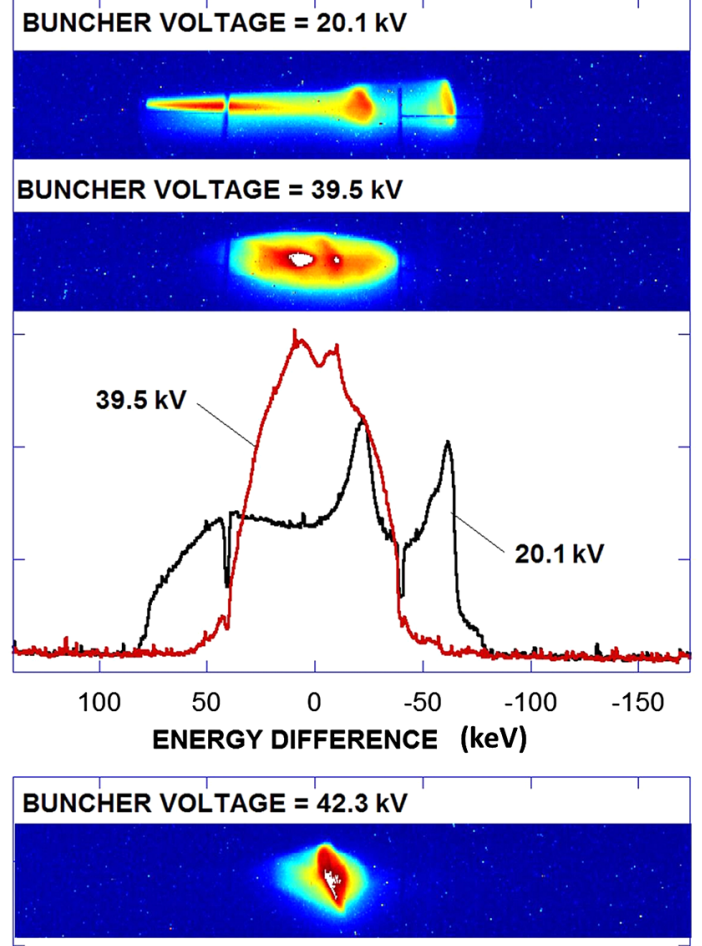

BUNCHER VOLTAGE $=\mathbf{5 0 . 2} \mathrm{kV}$
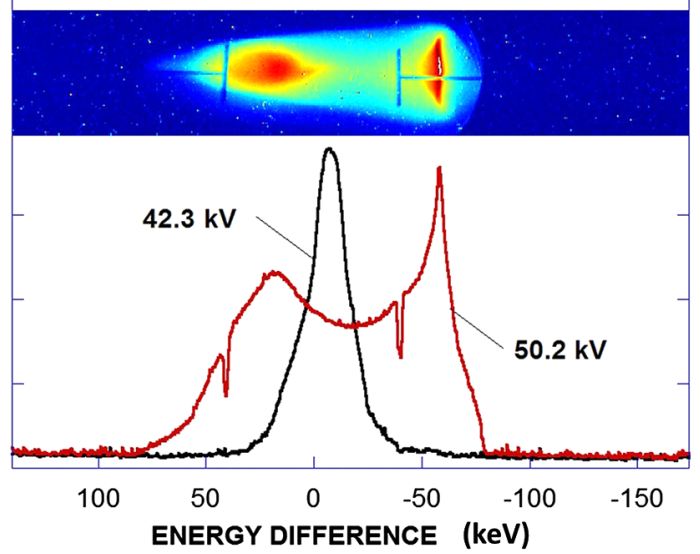

FIG. 4. Beam images on energy spectrometer screen YAG-05 and corresponding energy spectra at four different buncher voltages. dc gun voltage is $230 \mathrm{kV}$, and booster cavities $\mathrm{BC} 1$ and $\mathrm{BC} 2$ phases are set to $-10^{\circ}$ and $+40^{\circ}$, respectively. The second solenoid SOL-02 is set to the current of $2.20 \mathrm{~A}$.

given as a function of the buncher voltage at three different settings of the solenoid SOL-02. At the bottom of the curves, below $\sim 20 \mathrm{keV}$, the peaks cannot be easily distinguished, and the numbers are given as FWHM values instead. Note that there is a dependence of the energy difference on the solenoid strength, which is especially clear at higher values of $V_{b}$, above $\sim 45 \mathrm{kV}$. From computer simulations, the longitudinal crossover is expected to occur before the entrance of the booster at these higher buncher powers. This indicates the role of space-charge effects in longitudinal bunch formation.

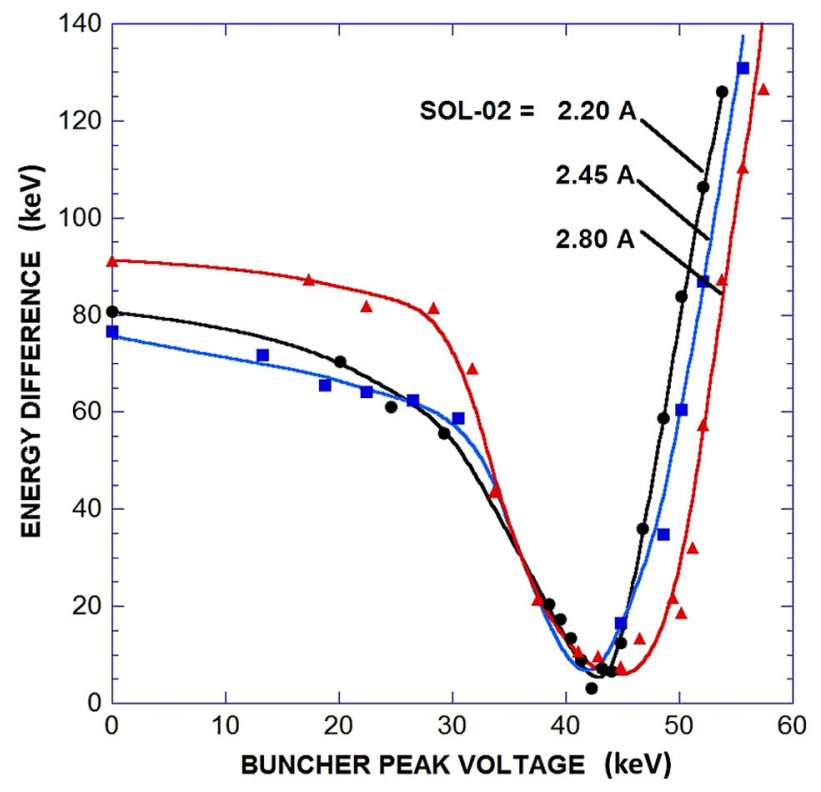

FIG. 5. The energy difference between two peaks in the beam energy spectra as a function of the buncher peak voltage and SOL-02 current. The BC2 phase is set to $+40^{\circ}$ here.

It is more informative to investigate the bunch structure if the second booster cavity $\mathrm{BC} 2$ is used purely as a streaking cavity. With $\mathrm{BC} 2$ set to $\mathrm{a}+90^{\circ}$ zero-cross phase, the energy spread $\Delta E$ of the bunch can be described by

$$
\Delta E=\sqrt{\Delta E_{0}^{2}+\left[\left(\frac{d E}{d z}\right)_{1}-\frac{d E_{2}}{d z}\right]^{2} \Delta z^{2}},
$$

where $\Delta E_{0}$ is a noncorrelated energy spread including all distortions of the longitudinal phase space, $(d E / d z)_{1}$ is the energy chirp of the bunch at the exit from $\mathrm{BC} 1$, and $\Delta z$ is the bunch length. The additional energy chirp introduced by $\mathrm{BC} 2$ is determined by $d E_{2} / d z=2 \pi E_{20} / \lambda$, where $E_{20}$ is the peak BC2 voltage and $\lambda$ is the rf wavelength $(231 \mathrm{~mm}$ at $1.3 \mathrm{GHz}$ ). We may apply the same equation to quantify the bunch structure by assuming that $\Delta E$ and $\Delta z$ are the energy difference and longitudinal distance between beamlets, respectively.

At sufficiently high $\mathrm{BC} 2$ gradients, the noncorrelated energy spread $\Delta E_{0}$ can be neglected, and Eq. (1) simplifies to $d(\Delta E) / d E_{20}=2 \pi \Delta z / \lambda$, from which the longitudinal separation between the beamlets can be determined. Such a dependence is shown in Fig. 6 at two buncher peak voltages, 44.9 and $50.2 \mathrm{kV}$. From the slopes of the straight line fits, the longitudinal separation between peaks is deduced to be $3.3 \mathrm{~mm}$ at a buncher voltage of $44.9 \mathrm{kV}$ and $9.0 \mathrm{~mm}$ at $50.2 \mathrm{kV}$ (corresponding FWHM bunch lengths are close but slightly higher than those longitudinal distances between peaks). An extrapolation to a zero $\mathrm{BC} 2$ voltage can also give an estimate of the initial energy separation between beams at the exit from BC1: 200 keV 


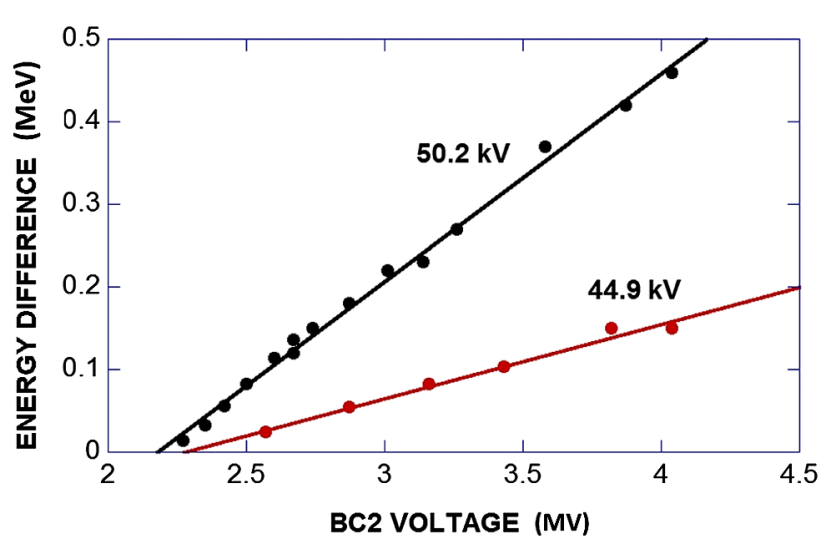

FIG. 6. The energy separation between beamlets as a function of the booster cavity BC2 peak voltage at two buncher voltages of 44.9 and $50.2 \mathrm{kV}$. Cavity BC2 is set to the streaking mode (zero-cross phase).

and $\sim 530 \mathrm{keV}$, respectively. The minimal postbooster energy spread condition, when both beamlets have approximately equal energy after exiting $\mathrm{BC} 2$, is achieved at a $\mathrm{BC} 2$ voltage of $\sim 2.3 \mathrm{MV}$.

The above measurements suggest that the electron bunch at the exit of the booster has a structure that includes two sufficiently well-defined beamlets: one at the head and the other at the tail of the bunch and separated longitudinally by a distance approximately equal to the full bunch length. Several different factors can potentially cause the development of such a structure, and they were investigated experimentally.

The "two-beamlets" structure remains present in energy spectra over a wide range of bunch charges, from $\sim 15 \mathrm{pC}$ up to $\sim 100 \mathrm{pC}$ (Fig. 7). The energy difference between the peaks does depend on the bunch charge, and this is likely to be the result of variations in the bunch length and energy chirp in the gun beam line, as both are space-charge dependent. These data were obtained by varying only

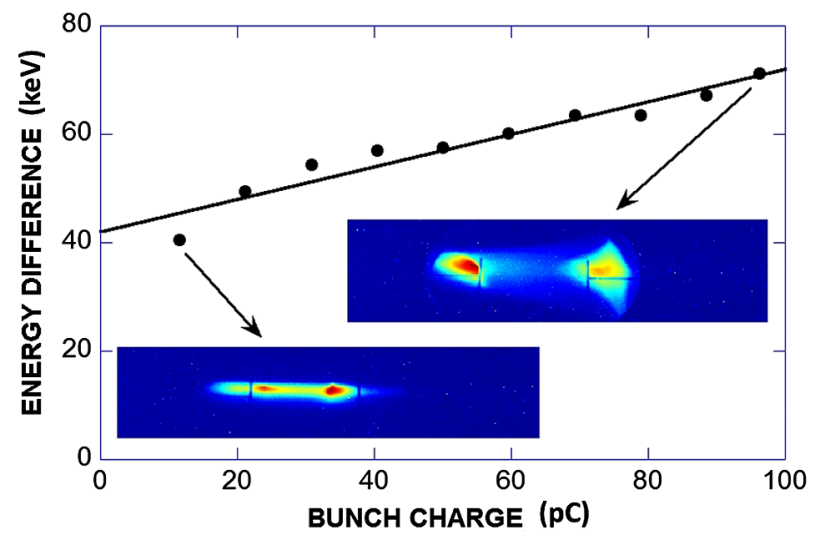

FIG. 7. The dependence of energy difference between beamlets on bunch charge. Corresponding beam images on energy spectrometer screen YAG-05 are shown for high and low bunch charges. the photoinjector laser power, all other machine settings remaining unchanged. The resulting variation in beam images on YAG-05 is illustrated by two extreme cases in Fig. 7, one of low bunch charge and one at high bunch charge. This experiment demonstrates that the electron space-charge forces might be a factor in the energy separation between two peaks but are not the main factor in developing the structure within the electron bunch.

Another potential factor could be the temporal structure of the laser pulse on the cathode of the dc gun. The ALICE photoinjector laser system employs a laser pulse stacker that combines four 7 ps FWHM pulses into one of 28 ps FWHM. The resulting laser pulse may not be necessarily uniformly flat, as demonstrated by measurements with a streak camera [14], and thus could be the cause of the resulting longitudinal structure of the electron bunch. However, an experiment with a fundamental Gaussian 7 ps long laser pulse (with the pulse stacker removed) clearly showed the same bunch structure as seen with the pulse stacker included.

The potential effect of quantum efficiency (QE) nonuniformity across the photocathode was also tested experimentally by moving the laser spot to areas of the photocathode surface with high degrees of $\mathrm{QE}$ uniformity. It was confirmed that the beam structure persisted.

The above experiments suggest that the beam structure formation is likely to be caused by complex beam dynamics in the SC booster during the initial stages of beam acceleration from a low gun voltage of a few hundred $\mathrm{keV}$.

The effect of the accelerating gradient in the first booster cavity $\mathrm{BC} 1$ was investigated in an experiment where the beam energy at the exit from $\mathrm{BC} 1$ was changed from 3.0 to $5.0 \mathrm{MeV}$ by varying the $\mathrm{BC} 1$ gradient. $\mathrm{BC} 2$ was set to $+90^{\circ}$ zero-cross phase for the middle of the range beam energy of 4.0 MeV. At different $\mathrm{BC} 1$ gradients, the beam energy at the $\mathrm{BC} 2$ exit was kept constant at $4.0 \mathrm{MeV}$ by adjusting the $\mathrm{BC} 2$ phase by up to $10^{\circ}$ around the zero-cross phase. This experimental procedure allowed simultaneously maintaining the energy chirp, introduced by $\mathrm{BC} 2$, and the mean beam energy while varying the $\mathrm{BC} 1$ gradient and minimizing any effects that would otherwise complicate the analysis of beam energy spectra. The spectra were measured at three different buncher voltages, and results are shown in Fig. 8 together with representative energy spectra at a buncher voltage of $44.9 \mathrm{kV}$.

Energy separation between beamlets (and the overall energy spread) reduces with an increasing $\mathrm{BC} 1$ gradient. This effect does not have a simple interpretation, because the $\mathrm{BC} 1$ gradient affects several different parameters of the electron bunch simultaneously, including the bunch length and the energy chirp at the exit of $\mathrm{BC} 1$. The effective accelerating $\mathrm{BC} 1 \mathrm{rf}$ phase also changes due to a different rate of phase slippage at the beginning of the acceleration. However, the main observation here is that the peaks at the ends of the energy spectra become narrower and more 

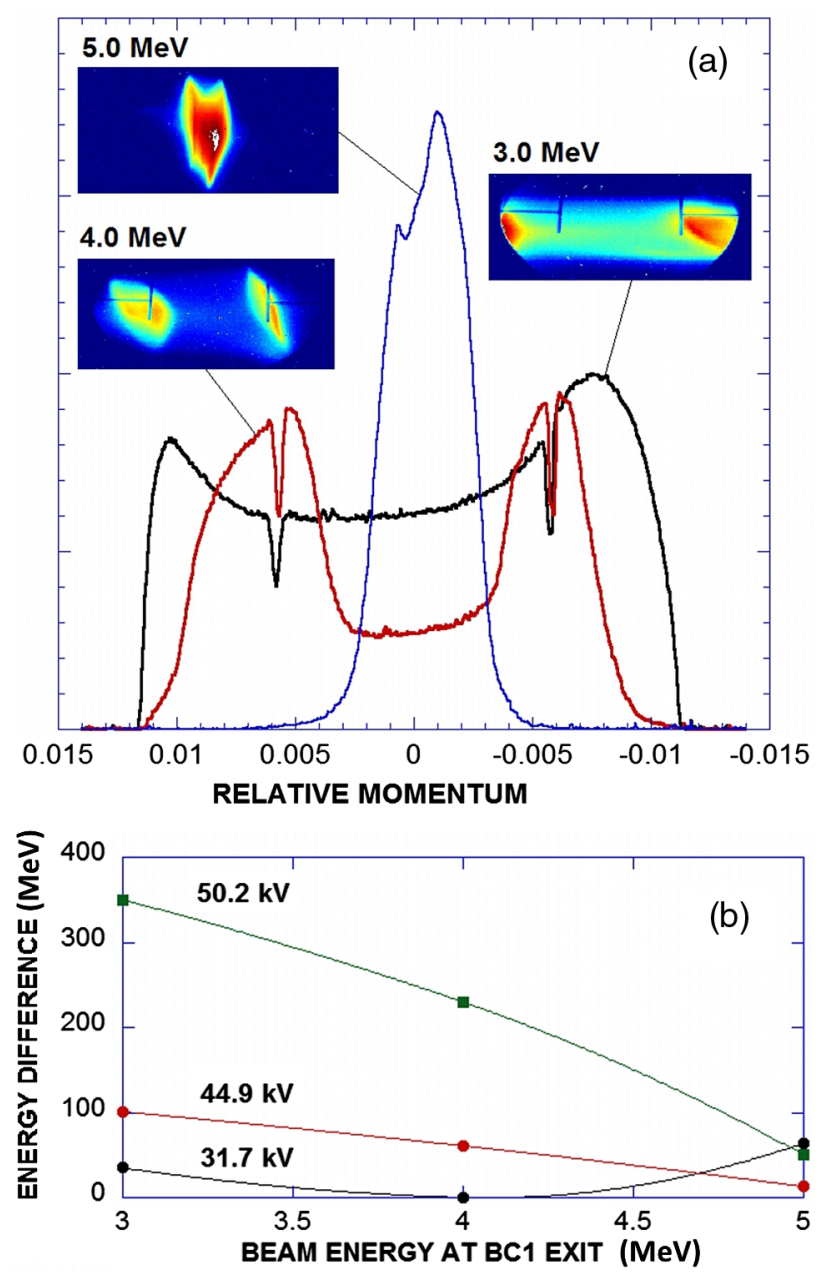

FIG. 8. The effect of the first booster cavity BC1 gradient on the beam energy spectrum. (a) Representative energy spectra at $V_{b}=44.9 \mathrm{kV}$. (b) Energy difference between beamlets as a function of the booster cavity BC1 voltage at three different buncher voltages. Data were collected at a constant beam energy of $4.0 \mathrm{MeV}$ at the exit from the booster second cavity $\mathrm{BC} 2$ and constant energy chirp introduced by $\mathrm{BC} 2$.

prominent with respect to the main body of the bunch when the $\mathrm{BC} 1$ gradient increases. This narrowing in the spectral peaks implies an increase in the proportion of the bunch charge located in the beamlets, thus making the bunch generally less uniform. This behavior corroborates the hypothesis that the observed beam structure is the result of a beam dynamics during the initial acceleration in the booster.

Several experiments were conducted to investigate the relation between the transverse beam structure, the beam core and halo, similar to that shown in Fig. 2(a), and the longitudinal beam structure.

Using higher than nominal buncher voltages, it was possible to accentuate the transverse beam structure to such an extent that the beam image acquires a crosslike shape on the YAG-04 screen in the middle of the dogleg section of the injector beam line [Fig. 9(b)]. With these machine

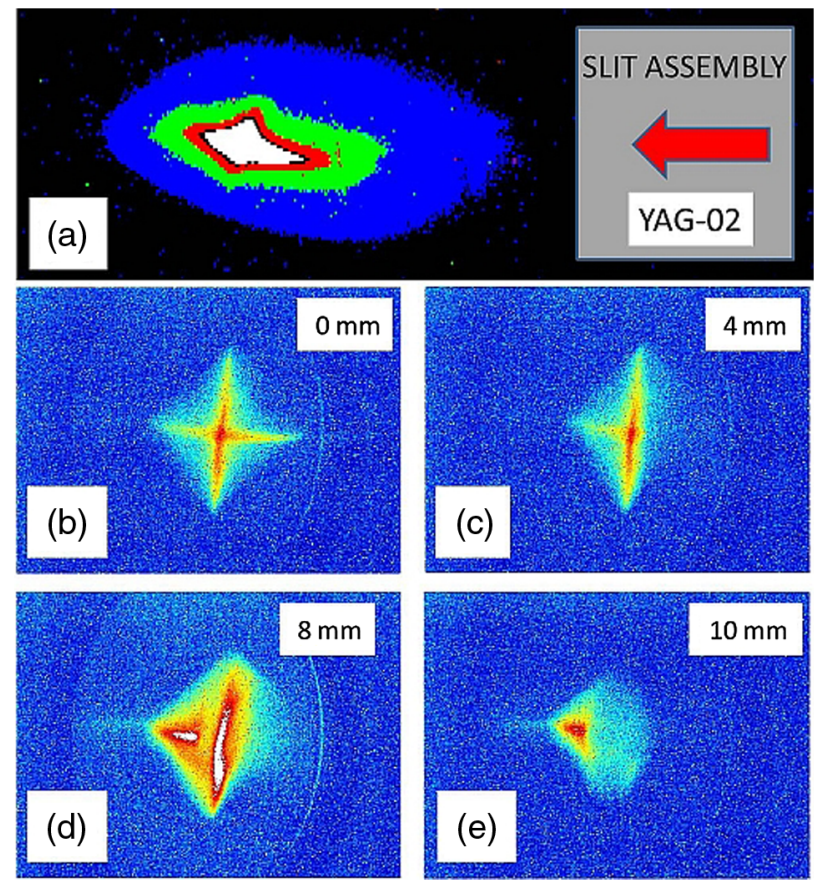

FIG. 9. (a) Beam image at the exit from the booster on the YAG-02 screen with nominal booster cavity phases of $-10^{\circ} /+25^{\circ}$, a beam energy of $6.5 \mathrm{MeV}$, but with a higher than nominal buncher voltage. The image is gamma-corrected to accentuate the core and the halo of the beam. (b)-(e) Four beam images on the YAG-04 screen in the middle of the injector dogleg while the beam at the YAG-02 position is progressively blocked from right to left with the slit assembly.

settings, the beam at the YAG-02 position was progressively blocked from right to left with a $1 \mathrm{~mm}$ thick copper slit assembly [Fig. 9(a)]. The resulting beam images on YAG-04 screen are shown in Figs. 9(c)-9(e). It was concluded from this set of images that the core of the beam on YAG-02 corresponds to the vertical part of the cross on YAG-04 and the halo to the horizontal part of the cross.

Using the same technique, but utilizing the YAG-03 slit assembly to progressively block the beam and analyzing the energy spectrum on the spectrometer screen YAG-05, the correlation between transverse and longitudinal features of the bunch were also investigated. $\mathrm{BC} 2$ was set to $+90^{\circ}$ zero-cross phase, and its gradient was chosen such that the energy chirp became negative at the exit of the booster; i.e., the head of the bunch had a lower energy than the tail. The longitudinal beam structure was again exaggerated by setting the buncher voltage to $50.2 \mathrm{kV}$, above the optimal level, and the intensities of the two peaks in the energy spectra were recorded as a function of the beam block position, shown in Fig. 10. The intensity of the lower energy peak (head of the bunch, red line) progressively decreases while the block moves into the beam. In contrast, the intensity of the higher energy peak (tail, black line) remains approximately constant until at least half of the beam is intercepted by the block. It was concluded from 


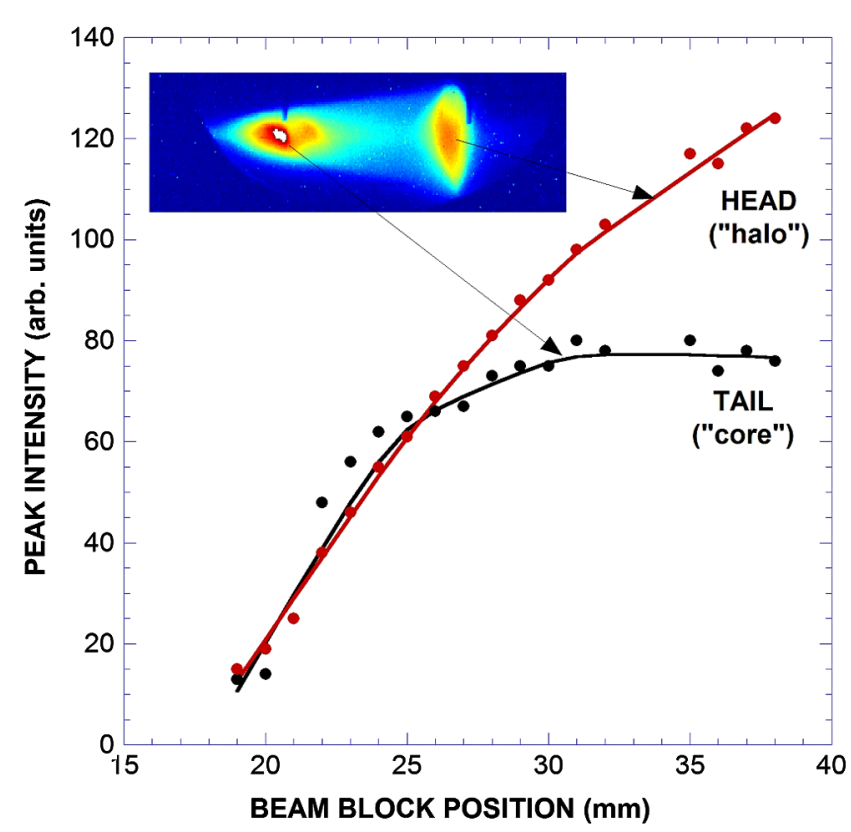

FIG. 10. Variation of the intensity of energy spectrum peaks while the beam is progressively blocked by a slit assembly located at the YAG-03 position.

these measurements that the large halolike part of the beam corresponds to the beamlet at the head of the bunch, and the corelike part of the beam forms the beamlet at the tail of the bunch.

The set of experimental data presented in this section leads us to the conclusion that, at a relatively low dc photoelectron gun voltage of $230 \mathrm{kV}$, the electron bunches have a structure consisting of two (and at some nonoptimal settings, three) beamlets. The two beamlets within the bunch are not only separated longitudinally but also have different electron energies and transverse phase space properties at the exit from the booster cavities.

\section{ELECTRON BUNCH STRUCTURE AT 325 KV GUN VOLTAGE}

Installation of a larger HV insulation ceramic on the ALICE photoinjector gun [6] enabled investigations of the
TABLE I. FWHM bunch parameters at $325 \mathrm{kV}$ gun voltage.

\begin{tabular}{lccc}
\hline \hline $\begin{array}{l}\text { Buncher peak } \\
\text { voltage }(\mathrm{kV})\end{array}$ & $\begin{array}{c}\text { Uncorrelated } \\
\text { energy spread } \\
(\mathrm{keV})\end{array}$ & $\begin{array}{c}\text { Energy chirp from } \\
\mathrm{BC1}(\mathrm{keV} / \mathrm{mm})\end{array}$ & $\begin{array}{c}\text { Bunch } \\
\text { length } \\
(\mathrm{mm})\end{array}$ \\
\hline 31.7 & 10 & 1.6 & 3.7 \\
54.9 & 5 & 20 & 2.5 \\
63.4 & 16.5 & 60 & 0.42 \\
\hline \hline
\end{tabular}

bunch structure at a higher gun voltage of $325 \mathrm{kV}$. At this gun voltage, the beam structure cannot be observed as easily as at $230 \mathrm{kV}$. However, the two-beamlets bunch structure in the energy spectrum could be induced by setting the injector to nonoptimal parameters, most notably by varying the buncher power as illustrated in Fig. 11. Transversely, no beamlet structure could be distinguished at any settings.

Measurements similar to those presented in Fig. 6 were repeated at a gun voltage of $325 \mathrm{kV}$ and shown in Fig. 12, with the difference that the FWHM widths of the energy spectra were recorded instead of the energy separation between the beams at the head and the tail of the bunch. The data were fitted using the analytical formula of (1), and the noncorrelated energy spread $\Delta E_{0}$, the energy chirp of the bunch at the exit from $\mathrm{BC} 1(d E / d z)_{1}$, and the FWHM bunch length $\Delta z$ were evaluated as a function of the buncher peak voltage. The results are summarized in Table I. Since BC2 was set to a zero-cross phase during these measurements, the beam energy here was constant at $4.0 \mathrm{MeV}$.

In measurements presented in Fig. 12, the bunch structure was clearly observed at a low buncher voltage of $V_{b}=31.7 \mathrm{kV}$ (as in Fig. 11), while it can hardly be seen at the approximately nominal buncher voltage of $V_{b}=54.9 \mathrm{kV}$ and cannot be distinguished at all at a slightly higher voltage of $V_{b}=63.4 \mathrm{kV}$. The latter is probably due to the fact that the bunch length is extremely small at this buncher power, which leads to both a much higher uncorrelated energy spread and an increased bunch energy chirp (Table I).
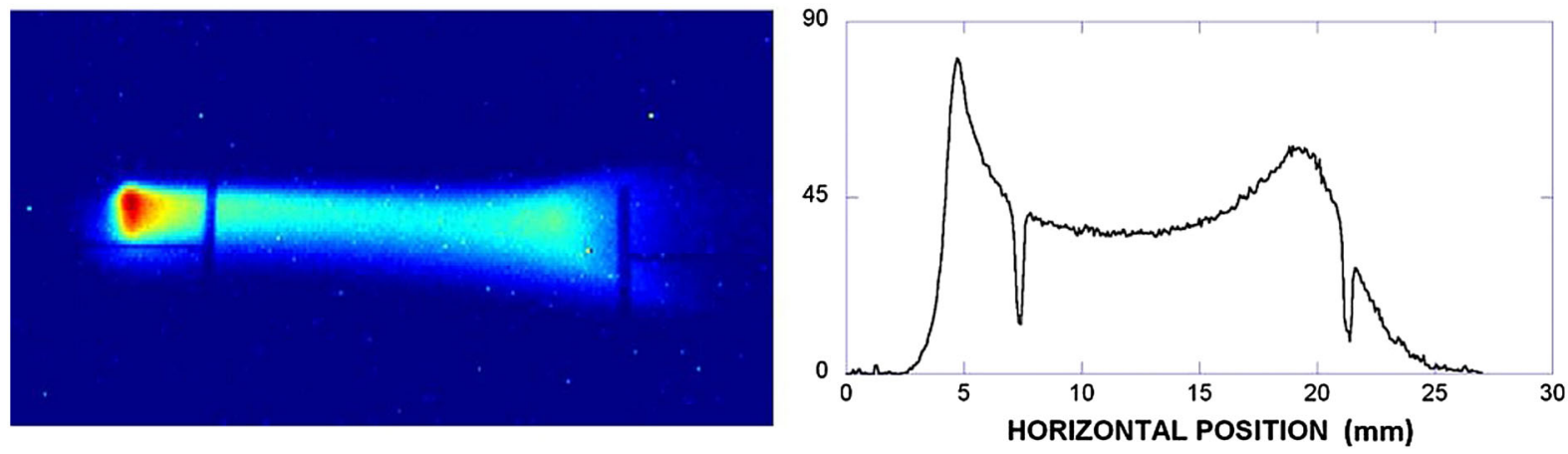

FIG. 11. Two-beamlets structure in an energy spectrum at $325 \mathrm{kV}$ gun voltage and a significantly reduced buncher voltage of $31.7 \mathrm{kV}$. 


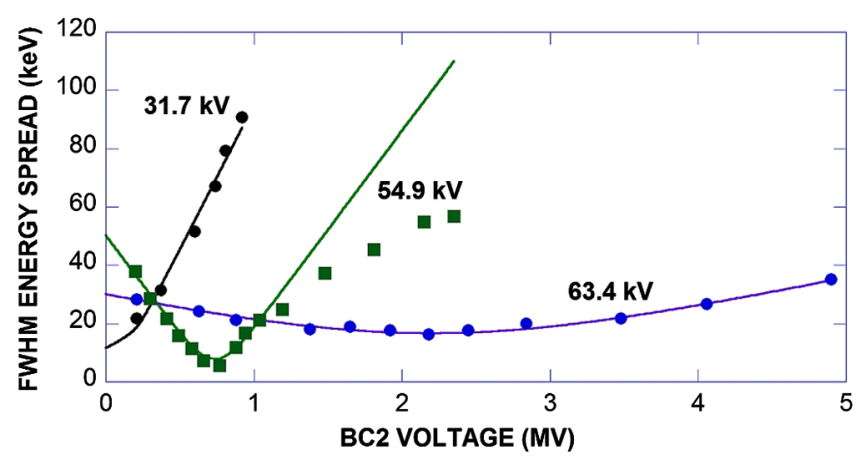

FIG. 12. FWHM energy spread as a function of the booster cavity BC2 peak voltage at three buncher voltages of 31.7, 54.9, and $63.4 \mathrm{kV}$. Cavity BC2 is set to the streaking mode (zero-cross phase). The gun voltage is $325 \mathrm{kV}$.

The overall beam quality is significantly improved at these higher gun voltages. This is also reflected in the lower emittances that can be achieved while optimizing the buncher power. This is illustrated in Fig. 13, where the emittance was measured using the slit at YAG-02 and the screen at YAG-03. The red dots in Fig. 13 represent the emittance measurements at a gun voltage of $325 \mathrm{kV}$ using the alternative quadrupole scan method in a simple quaddrift-screen configuration. All of these measurements were carried out using the standard injector setup at $6.5 \mathrm{MeV}$ beam energy after $\mathrm{BC} 2$.

\section{COMPUTER SIMULATION RESULTS}

At the gun voltage of $230 \mathrm{kV}$, the nominal buncher voltage was around $35 \mathrm{kV}$ at which the internal bunch structure was clearly visible, as presented in Fig. 2. However, this was difficult to investigate experimentally due to the shortness of the bunch. Therefore, most

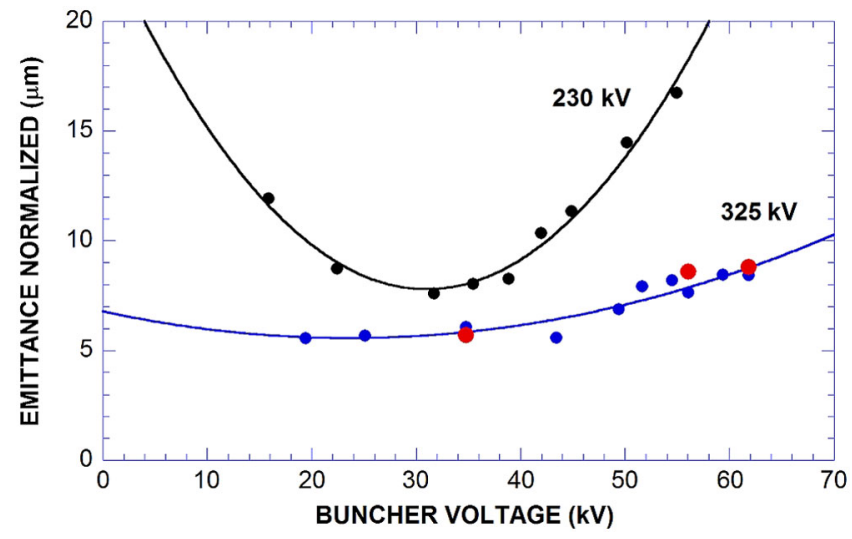

FIG. 13. Horizontal normalized emittance at the exit from the booster as a function of the buncher peak voltage at gun voltages of 230 (black) and $325 \mathrm{kV}$ (blue) using a slit-screen technique. The red dots represent the emittance measurement at $325 \mathrm{kV}$ by a quadrupole scan technique. measurements were made with either lower $(\sim 30 \mathrm{kV})$ or higher $(\sim 50 \mathrm{kV})$ buncher voltages.

ASTRA [9] simulation results for the bunch development in the case of the lower buncher voltage of $31.7 \mathrm{kV}$ were presented earlier in Fig. 3, and the corresponding bunch behavior was described in Sec. II. Note the strong longitudinal bunch compression in the first cell of the first booster cavity $\mathrm{BC} 1$. The simulated bunch development at the higher buncher voltage of $V_{b}=50.2 \mathrm{kV}$ is shown in Fig. 14. Here the bunch experiences a longitudinal crossover before reaching the booster, and the first cell of $\mathrm{BC} 1$ does little for the bunch compression.

Dividing the bunch into several longitudinal slices, just after the rf buncher, and "painting" these slices in different colors allows the investigation of the development of the longitudinal bunch structure. In both cases of bunch compression, the longitudinal phase space undergoes a violent transformation either near the longitudinal crossover (high buncher voltage) or in the first cell of booster cavity $\mathrm{BC} 1$ (low buncher voltage). This takes the form of a nonlinear motion of longitudinal slices with respect to each other, resulting in an overlap of the longitudinal slices. In simulations, this is especially notable at the head of
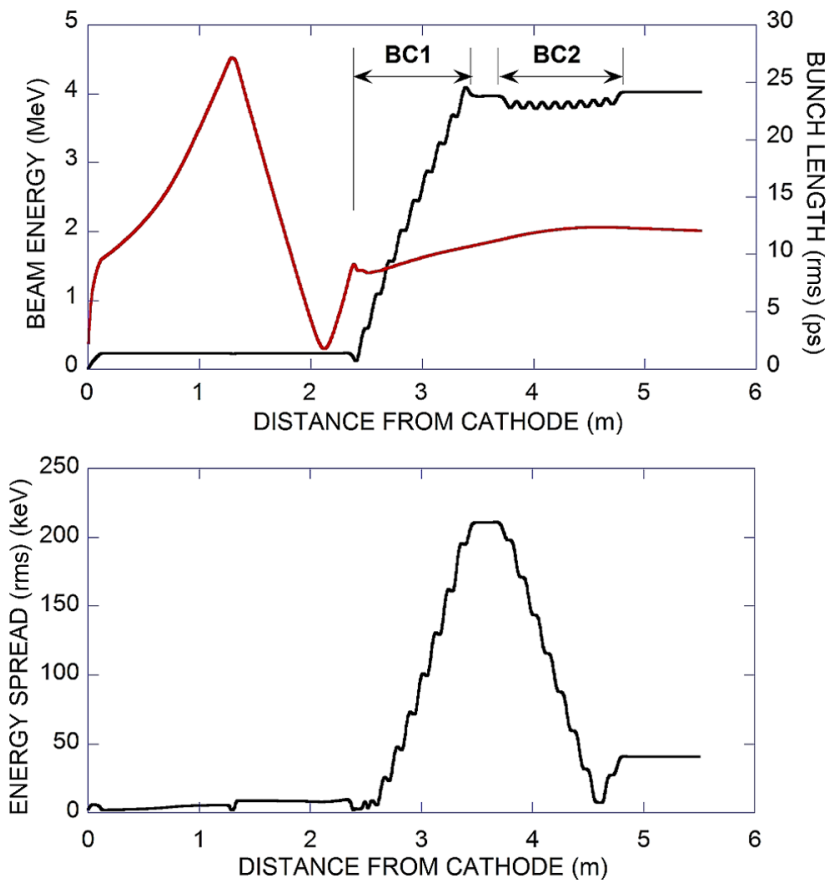

FIG. 14. Simulated (ASTRA) development of the mean beam energy, the rms bunch length, and the bunch energy spread from the photoinjector cathode to the exit from the booster. The buncher voltage is $50.2 \mathrm{kV}$, thus causing the longitudinal crossover before the booster. The first cavity $(\mathrm{BC} 1)$ is set to $-10^{\circ}$ offcrest phase. The $\mathrm{BC} 2$ cavity is set to $+90^{\circ}$ zero-cross phase. The $\mathrm{BC} 2$ gradient is chosen such that the longitudinal phase space is rotated to the negative energy chirp at the exit from $\mathrm{BC} 2$. The gun voltage is $230 \mathrm{kV}$, and the final beam energy is $4.0 \mathrm{MeV}$. 
the bunch but less so at the tail. The overlap creates irregularities in the longitudinal current profile that, during further bunch acceleration in the booster cavities, manifest themselves in irregularities of the energy spectra.

At buncher voltages of $40-45 \mathrm{kV}$, the simulations show that the adjacent longitudinal slices heavily overlap each other, creating very irregular current distributions and energy spectra. The bunch length at these buncher voltages is also the shortest at $\sim 2$ ps rms. Such short bunches could not be streaked out efficiently by the $\mathrm{BC} 2$ gradient to ensure sufficient energy resolution and therefore were not investigated experimentally.

The simulated bunch properties at the exit from the booster for two special cases of a lower buncher voltage of $31.7 \mathrm{kV}$ and a higher voltage of $50.2 \mathrm{kV}$ are presented in Fig. 15. These buncher voltages and other injector settings are the same as those used in the simulation results shown in Figs. 3 and 14. The development of the longitudinal phase space from the exit from the buncher to the exit from the first booster cavity $\mathrm{BC} 1$ for the case of $31.7 \mathrm{kV}$ buncher voltage is illustrated in Fig. 16. The peaks at the head of the bunch are readily identifiable in the longitudinal current distributions and energy spectra (Fig. 15). They are more pronounced at a higher buncher voltage $\left(V_{b}\right)$ (black lines) but are also present at lower buncher voltages (red lines). This is the result of a complex structure of the longitudinal phase space at the head of the bunch (Fig. 15). ASTRA simulations do not show such prominent features at the tail of the bunch, especially at lower buncher voltages. This is in contrast to experimental observations, where the two peaks in the energy spectra were observed within the whole range of buncher voltages investigated (see, for example, Fig. 4). It has to be noted, however, that in experiments the peaks at the tail of the bunch were normally much broader and with lower amplitude compared to the peaks at the head of the bunch. The discrepancy between the experiment and simulations may be caused by complex physical processes governing the bunch formation during the initial acceleration in the booster and that are not fully encompassed by the computer simulation model so far. The current model does not include the effects of wakefields and uses a simplified 1D field map for the booster where the off-axis field is calculated by extrapolation. An important factor could be a misalignment between the beam and the booster axis that is not accounted for in the model. The list of mechanisms could be continued but, currently, is mostly speculative and is a subject of further specific investigation. The number of macroparticles and grid meshing in the simulations also are important factors. As an example, increasing the number of macroparticles from 10000 to 30000 (at which all the simulations in this paper were conducted) does not appreciably change the integral characteristics of the bunch (e.g., rms emittance, energy spread, and bunch length) but significantly changes the internal structure of the longitudinal phase space.
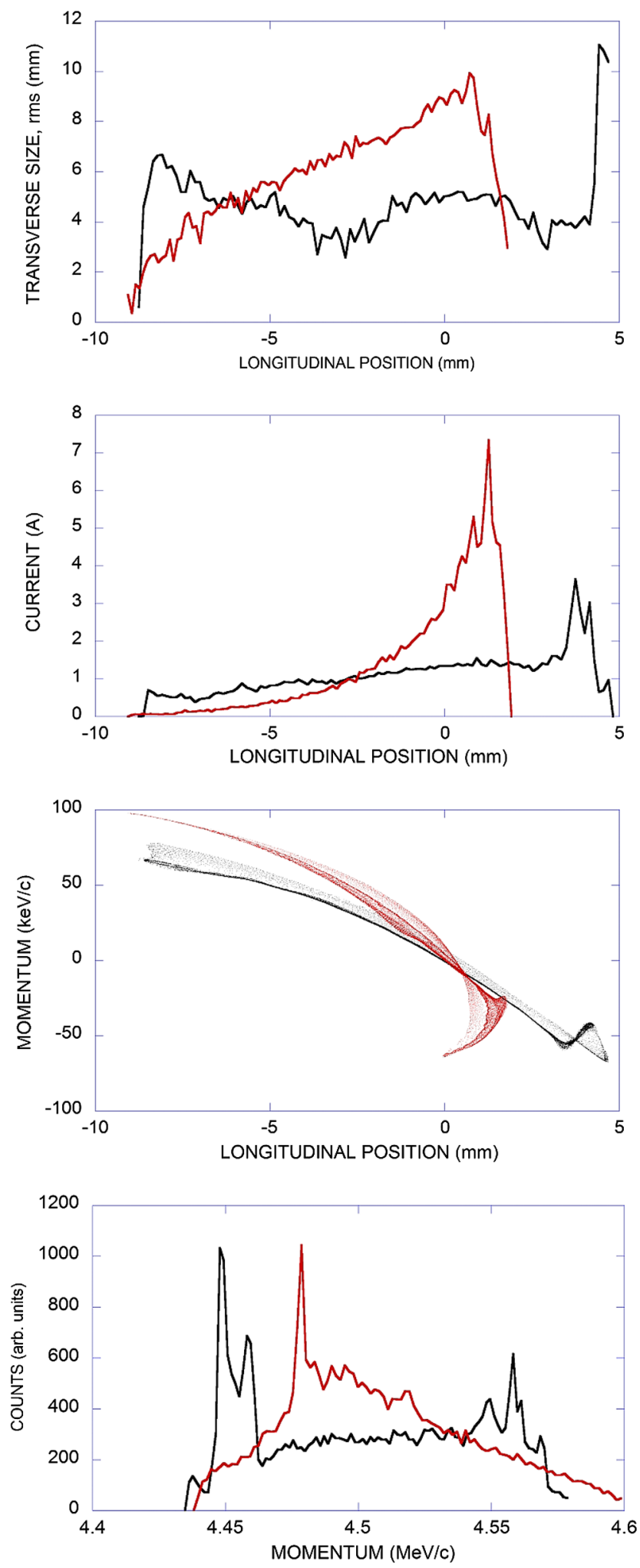

FIG. 15. Electron bunch distributions at the dc gun voltage of $230 \mathrm{kV}$ and two buncher voltages of 31.7 (red) and $50.2 \mathrm{kV}$ (black). The distributions are shown at the exit from the booster. From top to bottom: rms transverse beam size and current distributions along the bunch, longitudinal phase space, and energy spectra. The head of the bunch is at positive values of the longitudinal position. 

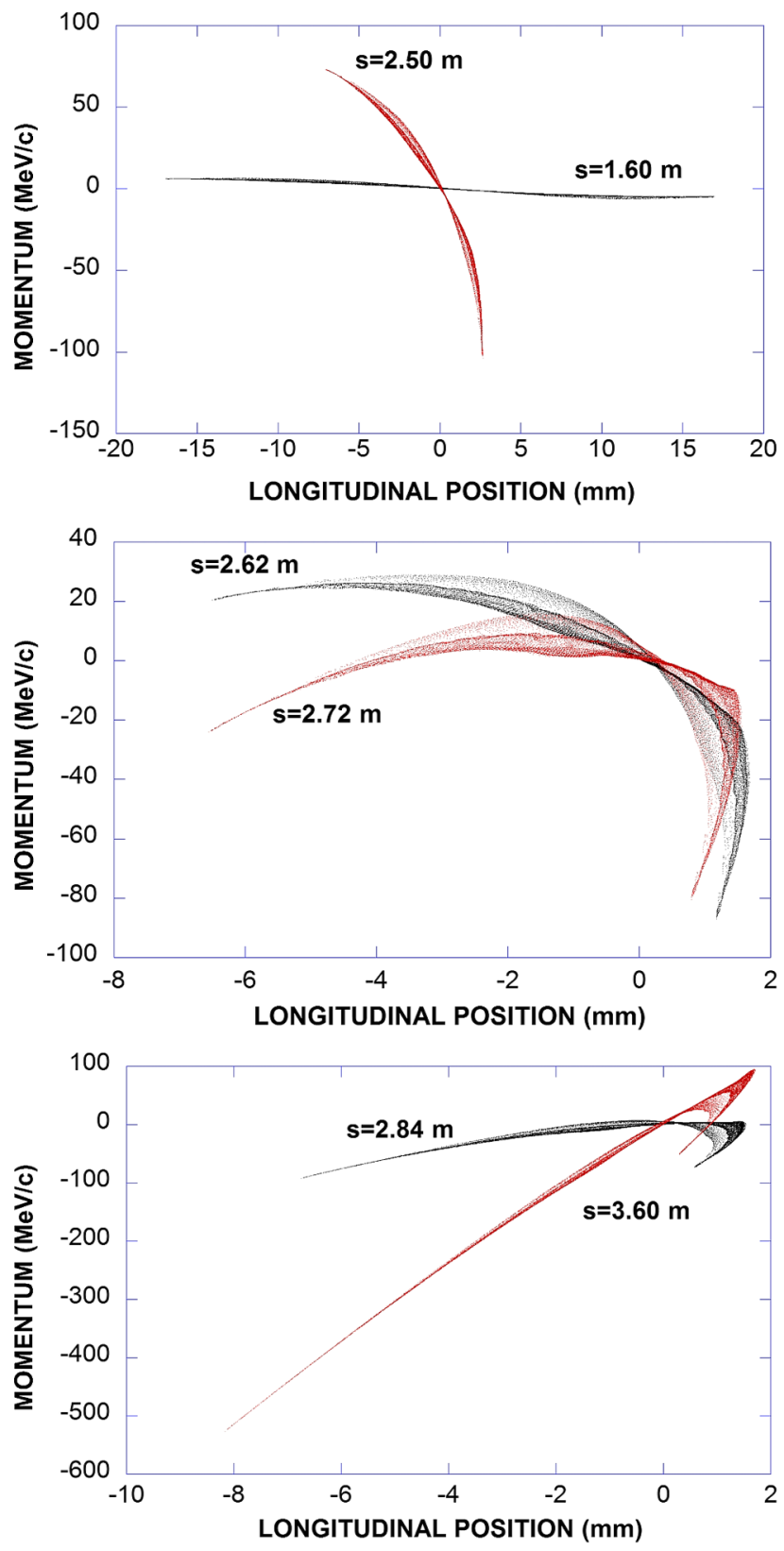

FIG. 16. Evolution of the longitudinal phase space at $230 \mathrm{kV} \mathrm{dc}$ gun voltage and $31.7 \mathrm{kV}$ peak buncher voltage (parameters correspond to Fig. 3). Distributions are shown at the exits from the buncher $(s=1.60 \mathrm{~m})$, the first four cells of BC1 $(s=2.50$, $2.62,2.72$, and $2.84 \mathrm{~m})$, and the last cell of BC1 $(s=3.60 \mathrm{~m})$. The phase space at the exit of BC2 is shown in Fig. 15. The head of the bunch is at positive values of the longitudinal position.

No bunch structure was evident in ASTRA simulations within a full range of buncher voltages at the gun voltage of $325 \mathrm{kV}$, in agreement with experimental observations. As expected, the longitudinal bunch slices do move in the longitudinal phase space during strong bunch compression in the first cell of $\mathrm{BC} 1$, but they do not overlap each other.

We may compare the simulated longitudinal phase space development in the ALICE injector (Figs. 15 and 16) with that of the Cornell injector [15-17], which also employs a dc HV photoelectron gun and a SC linac. Unlike ALICE, the Cornell SC linac has five two-cell cavities with independent settings of accelerating gradients and phases. The beam evolution in the Cornell injector can be found in Ref. [18] for the case of $500 \mathrm{kV}$ gun voltage. Longitudinal phase space development from the dc gun cathode to the entrance of the linac is qualitatively very similar in both injectors. Further acceleration in the linac exhibits, however, several distinct differences. In the ALICE injector, a sharp longitudinal compression of the bunch takes place over a short distance in the first cell of the first cavity BC1 (Fig. 3). The negative energy chirp imposed by the buncher is initially increased sharply but then gradually transformed to positive towards the exit from BC1 (Fig. 16). The BC2 can control the chirp in a wide range including making the chirp negative as shown in Fig. 15. In contrast, the simulations of the Cornell injector [18] show less abrupt bunching in the beginning of the booster that takes place over the first two (out of five) booster cavities. The energy chirp remains negative throughout acceleration. The overlapping of the longitudinal bunch slices is much smaller than in ALICE although still evident. It is reasonable to suggest that the differences in behavior are due to higher gun voltage and more "distributed" acceleration in the case of the Cornell injector.

\section{SUMMARY}

The results presented here suggest that the two-beamlets substructure within the electron bunch generated by a dc HV photoelectron gun develops due to the low energy beam dynamics of the injector, before the second booster cavity. The structure is characterized by the existence of two beamlets, one at the head and one at the tail of the bunch.

Conclusions made from experimental measurements combined with ASTRA simulations suggest the following scenario for the development of the bunch electron structure: Two peaks in the longitudinal bunch profile are formed as a result of strong velocity bunching when the longitudinal bunch slices move nonlinearly with respect to each other-adjacent slices overlap, thus forming the current peaks at the head and the tail of the bunch. This effect takes place either in the first cell of the first booster cavity $\mathrm{BC} 1$ (lower buncher voltages) or during a longitudinal crossover before $\mathrm{BC} 1$ (higher buncher voltages). Upon further acceleration at off-crest cavity phases, this longitudinal profile is transformed into a similar energy spectrum with two peaks at low and high energies. Spacecharge forces play a noticeable, but nondominant, role in this process.

It has to be noted that, although the simulations do show processes that would lead to a development of the twobeamlets structure within the bunch, the simulations results are not an exact replica of experimental results. This is 
likely to be due to limitations in the computer model to accurately describe the complex mixture of physical processes taking place during the initial stages of acceleration in the rf booster.

Measurements presented in Figs. 2 and 9 demonstrate that, apart from a difference in the electron energy, the beamlets also have different transverse properties and hence cannot be controlled simultaneously in the same lattice. This could be a strong contributing factor to the irregular beam shapes observed on different screens around the machine; see, for example, Fig. 2(c).

At an increased gun voltage (in this work, $325 \mathrm{kV}$ as compared to $230 \mathrm{kV}$ ) the beam structure is much less pronounced and could be identified only at nonoptimal injector settings.

Experimental data also suggest that lower gradients in the first booster cavity could be beneficial in terms of preserving the longitudinal quality of the electron bunch, as demonstrated in Fig. 8. Ideally, the booster should be designed as a multicavity linac with individual control of the gradients and accelerating phases in each cavity, similar to what has been realized in the Cornell photoinjector [15-18].

In conclusion, the bunch structure investigated in this paper is likely to be a common feature of electron beam injectors with relatively low energy electron guns (HV dc photoelectron guns, in particular). This unwanted structure can be alleviated by increasing the gun voltage to as high a level as practically possible and by controlling the beam acceleration in the first accelerating structure.

[1] S. Benson et al., in Proceedings of the 22nd Particle Accelerator Conference, PAC-2007, Albuquerque, NM (IEEE, New York, 2007), pp. 79-81, http://accelconf .web.cern.ch/AccelConf/p07/PAPERS/MOOAAB03.PDF.

[2] P. Evtushenko, S. Benson, G. Biallas, J. Coleman, C. Dickover, D. Douglas, M. Marchlik, D. Sexton, and C. Tennant, in Proceedings of the 24th Particle Accelerator Conference, PAC-2011, New York, 2011 (IEEE, New York, 2011), pp. 1446-1448, http://accelconf.web .cern.ch/AccelConf/PAC2011/papers/weocn4.pdf.

[3] D. Douglas, in Proceedings of the 14th Beam Instrumentation Workshop, Santa Fe, NM (LANL, Los Alamos, 2010), pp. 506-515, http://accelconf.web.cern.ch/ AccelConf/BIW2010/papers/weimnb02.pdf.

[4] S. L. Smith et al., Optic issues in ongoing ERL projects, Nucl. Instrum. Methods Phys. Res., Sect. A 557, 145 (2006).
[5] F. Jackson et al., in Proceedings of the 2nd International Particle Accelerator Conference, San Sebastián, Spain (EPS-AG, Spain, 2011), pp. 934-936, http://accelconf.web .cern.ch/AccelConf/IPAC2011/papers/tuoda03.pdf.

[6] Y.M. Saveliev, in Proceedings of the 3rd International Particle Accelerator Conference, New Orleans, LA, 2012 (IEEE, Piscataway, NJ, 2012), pp. 613-615, http:// accelconf.web.cern.ch/AccelConf/IPAC2012/papers/ moppp022.pdf.

[7] N. R. Thompson, D. J. Dunning, J. A. Clarke, M. Surman, A. D. Smith, Y. Saveliev, and S. Leonard, First lasing of the ALICE infra-red Free-Electron Laser, Nucl. Instrum. Methods Phys. Res., Sect. A 680, 117 (2012).

[8] Y. M. Saveliev, D. J. Holder, B. D. Muratori, and S. L. Smith, in Proceedings of the 11th European Particle Accelerator Conference, Genoa, 2008 (EPS-AG, Genoa, Italy, 2008), pp. 208-210, http://accelconf.web.cern.ch/ AccelConf/e08/papers/mopc062.pdf.

[9] http://www.desy.de/ mpyflo/.

[10] C. Hernandez-Garcia et al., in Proceedings of FEL2004 Conference (Comitato Conferenze Elettra, Trieste, Italy, 2004), pp. 363-366, http://accelconf.web.cern.ch/ AccelConf/f04/papers/TUBOS02/TUBOS02.PDF.

[11] Handbook of Accelerator Physics and Engineering, edited by A. W. Chao and M. Tigner (World Scientific Publishing, Singapore, 2002), p. 106.

[12] C. E. Clayton and L. Serafini, Generation and transport of ultrashort phase-locked electron bunches to a plasma beatwave accelerator, IEEE Trans. Plasma Sci. 24, 400 (1996).

[13] L. Serafini, R. Zhang, and C. Pellegrini, Generation of subpicosecond electron bunches from RF photoinjectors, Nucl. Instrum. Methods Phys. Res., Sect. A 387, 305 (1997).

[14] Y. M. Saveliev, S. P. Jamison, L. B. Jones, and B. D. Muratori, in Proceedings of the 11th European Particle Accelerator Conference, Genoa, 2008 (EPS-AG, Genoa, Italy, 2008), pp. 211-213, http://accelconf.web.cern.ch/ AccelConf/e08/papers/mopc063.pdf.

[15] A. Bartnik, C. Gulliford, I. Bazarov, L. Cultera, and Bruce Dunham, Operational experience with nanocoulomb bunch charges in the Cornell photoinjector, Phys. Rev. ST Accel. Beams 18, 083401 (2015).

[16] I. V. Bazarov and C. K. Sinclair, Multivariate optimization of a high brightness dc gun photoinjector, Phys. Rev. ST Accel. Beams 8, 034202 (2005).

[17] C. Gulliford et al., Demonstration of low emittance in the Cornell energy recovery linac injector prototype, Phys. Rev. ST Accel. Beams 16, 073401 (2013).

[18] Animation of the beam motion in the Cornell injector: http://www.lepp.cornell.edu/ ib38/injector. 